\title{
Prediction of various chemical parameters of olive oils with Fourier transform infrared spectroscopy
}

\author{
Oguz Uncu, Banu Ozen* \\ Izmir Institute of Technology, Department of Food Engineering, Urla-Izmir, Turkey
}

\section{A R T I C L E I N F O}

\section{Article history:}

Received 22 December 2014

Received in revised form

27 April 2015

Accepted 2 May 2015

Available online 12 May 2015

\section{Keywords:}

Olive oil

Partial least square analysis

FTIR

Quality parameters

Oxidative stability

\begin{abstract}
A B S T R A C T
Vibrational spectroscopic techniques offer advantages such as rapid and accurate measurements with minimum sample preparation and waste generation. In this study, it was aimed at determining some important quality parameters (oxidative stability, colour pigments, fatty acid profile and phenolic composition) of olive oils by Fourier transform infrared spectroscopy as one of the vibrational spectroscopic methods. Partial least square calibration models were constructed in order to reveal any correlation between quality parameters and spectral data. Regression coefficients for developed models showed that oxidative stability (0.99), chlorophyll content (0.98), some major fatty acids (palmitic (0.87), oleic (0.94), and linoleic acids (0.97), saturated (0.91), monounsaturated (0.94) and polyunsaturated fatty acids (0.97)), hydroxytyrosol as a phenolic compound (0.97) and total phenolic content (0.99) were predicted successfully. Variable influence on the projection values indicated that palmitic, vanillic and cinnamic acids and hydroxytyrosol are the most significant contributors to oxidative stability of olive oils.
\end{abstract} (C) 2015 Elsevier Ltd. All rights reserved.

\section{Introduction}

Olive oil, extracted from the fruit of olive tree, is known for its precious nutritional, functional and sensorial qualities. Olive oil consumption has been increasing in recent years due to its positive health effects that are attributed to its balanced unsaturated fatty acid content and the presence of other functional compounds such as phenolics, tocopherols and chlorophyll (Matos et al., 2007; Temime et al., 2008). Extra virgin olive oil is defined as the oil which is produced only by mechanical processes like crushing, malaxation and centrifugation without any further chemical treatment. Since no refinement process is involved in its production, organoleptic and nutritional values of olive oils are well preserved as well as its defense mechanism against oxidative stress (Perona, Cabello-Moruno, \& Ruiz-Gutierrez, 2006). There are many quality parameters of olive oils that need to be monitored in order to assure organoleptic and sensorial properties of the final product. One of these parameters is oxidative stability which can provide an idea about the storage history of olive oil. Furthermore, major components like fatty acid profile and minor components such as polyphenol content and chlorophyll level are also considered as

\footnotetext{
* Corresponding author. Tel.: +90 232750 6319; fax: +90 2327506196

E-mail address: banuozen@iyte.edu.tr (B. Ozen).
}

important contributors to organoleptic and quality properties of olive oil (Mailer, 2004). Therefore, it is important to determine these parameters in a fast and a reliable way. For this purpose, spectroscopic methods like near infrared (NIR), mid-infrared (MIR), Raman and NMR have been used in several studies and they have advantages compared to time-consuming and expensive traditional methods since several analyses could be performed simultaneously with minimum waste generation (Moros, Garrigues, \& de la Guardia, 2010). For instance, high-resolution ${ }^{13} \mathrm{C}$ NMR was used to predict oxidative stabilities of different oils including olive oil successfully (Hidalgo, Gómez, Navarro, \& Zamora, 2002). Acidity and peroxide index of different types of edible oils were evaluated by NIR spectroscopy in another study (Armenta, Garrigues, \& de la Guardia, 2007). Oxidized fatty acid concentration under different oxidative status was determined with FTIR (Fourier transform infrared) spectroscopy in a study by Lerma-García, Simó-Alfonso, Bendini, and Cerretani (2011). Also, Raman spectroscopy has been recently used in monitoring fatty acid composition of different vegetable oils with promising results (Dong, Zhang, Zhang, \& Wang, 2013).

In the literature, FTIR spectroscopy has been mainly used in classification studies. Moreover, it has also gained popularity on the quantitative analysis due to the fact that the emitted IR energy is directly proportional to the concentration of compounds that are present in a tested sample (Ismail, van de Voort, \& Sedman, 1997). 
FTIR spectroscopy has already been used in peroxide value determination for different vegetable oils (Allendorf, Subramanian, \& Rodriguez-Saona, 2012) and in the quantification of fatty acids and triacylglycerols of olive oils (Galtier et al., 2008).

The aim of the present study is to investigate the ability of FTIR spectroscopy as a fast and a reliable method in the prediction of some important quality parameters of olive oils, oxidative stability, colour pigments (chlorophyll and carotenoid), fatty acid profile and phenolic compounds. Moreover, the effect of each measured chemical constituent on oxidative stability is evaluated.

\section{Materials and methods}

\subsection{Olive oil samples}

Sixty four olive oil samples were obtained from the various parts of Karaburun Peninsula of Izmir. Oils were extracted with an industrial scale two phase decanter system (Polat Machinery, Turkey) capable of processing 1.66 tonnes olive/h and located in Izmir Institute of Technology Campus and Eglenhoca village of Izmir. Samples in glass containers were kept in the dark at refrigeration temperature $\left(8^{\circ} \mathrm{C}\right)$ after their head spaces were flushed with nitrogen.

\subsection{Chemical reagents}

All reagents used in the experiments were of analytical grade and they were obtained from Riedel-de Haën (Germany), Sigma-Aldrich (Germany) and Merck (Germany). Phenolic acids (vanillic, syringic, caffeic, p-coumaric, o-coumaric, cinnamic, 4hydroxyphenyl acetic, 3-hydroxyphenyl acetic and 2, 3dihydroxybenzoic acids), flavonoids (apigenin, luteolin and vanillin) and phenolic alcohols (tyrosol and hydroxytyrosol) for HPLC analysis were the commercial phenolic standards (Fluka and Extrasynthase). Fatty acid methyl ester (FAME) mixture containing C4-C24 (2-4\% relative concentration) was used as a reference standard (Supelco \# 47885-U) for GC analysis.

\subsection{Chemical analyses}

\subsubsection{Oxidative stability (OS)}

Oxidative stability was determined with Rancimat equipment (873 Biodiesel, Metrohm, Switzerland) in terms of hour. Temperature range of this equipment is $50-220^{\circ} \mathrm{C}$ and temperature stability is less than $0.1^{\circ} \mathrm{C} .3 \mathrm{~g}$ of olive oil was placed inside the glass reaction vessel for the measurement. Carrier medium was selected as deionized water. Reaction temperature was set to a constant value of $120{ }^{\circ} \mathrm{C}$ for both columns of Rancimat apparatus with a constant $20 \mathrm{~L} / \mathrm{h}$ air flow.

\subsubsection{Total phenol content (TPC)}

Folin-Ciocalteu spectrophotometric method was used to determine the total amount of phenolic compounds in the olive oil samples (Montedoro, Servili, Baldioli, \& Miniati, 1992). All the results were calculated in terms of gallic acid (GA) as $\mathrm{mg} \mathrm{GA} / \mathrm{kg}$ oil using gallic acid standard curve. The measurements were repeated for two times for the extracted samples.

\subsubsection{High performance liquid chromatography (HPLC) analysis of phenolic compounds \\ The procedure from Brenes, García, García, Rios, and Garrido (1999) was used to extract phenolic compounds from olive oil samples. The extract having gallic acid as an internal standard was immediately injected to HPLC.}

Amounts of individual phenolic compounds in olive oil were determined by an HPLC (Agilent 1200 HPLC, USA) equipped with refractive index (RI) and photodiode array (DAD) detectors, an auto sampler (ALS G1329A) and a column oven. A C18 column (250*4 mm, $5 \mu \mathrm{m}$, SGE 8211, Australia) was used in analyses. Column temperature was kept at $35{ }^{\circ} \mathrm{C}$ and injection volume was $20 \mu \mathrm{L}$. Flow rate was adjusted to $1 \mathrm{~mL} / \mathrm{min}$. Two different mobile phases were used as water/acetic acid (99.8:0.2 v/v) and methanol. Initial concentrations of mobile phases were $90 \%$ for water/acetic acid and $10 \%$ for methanol. Concentration of mobile phases was adjusted over time by the following procedure; firstly, the concentration of methanol was increased to $30 \%$ in 10 min and kept there for $20 \mathrm{~min}$ and at the same time water/acetic acid concentration was decreased to $70 \%$. Then, methanol percentage was increased to $40 \%$ in $10 \mathrm{~min}$, kept for another $5 \mathrm{~min}$, followed by rising up to $50 \%$ in $5 \mathrm{~min}$, and kept for $5 \mathrm{~min}$. At last, methanol was increased to 60,70 , and $100 \%$ in 5 min periods. Finally, initial conditions were attained at the end of $85 \mathrm{~min}$.

Internal standard method was used in order to compensate any loss of phenolic compounds during the experimental procedures. Gallic acid was chosen as the internal standard. Major phenolic compounds found in olive oil were determined by using their commercial standard forms at two different wavelengths of 280 and $320 \mathrm{~nm}$. 5-point calibration curves for each standard were plotted and the results were expressed in terms of $\mathrm{mg} / \mathrm{kg}$.

\subsubsection{Chlorophyll \& carotenoid measurement}

Chlorophyll and carotenoid contents of olive oils were determined according to a procedure in literature (Mínguez-Mosquera, Rejano-Navarro, Gandul-Rojas, Sánchez Gómez, \& GarridoFernandez, 1991). $7.5 \mathrm{~g}$ of an olive oil sample was weighted into a test tube and filled up to $25 \mathrm{~mL}$ with cyclohexane. The absorbance corresponding to chlorophyll and carotenoid fractions were measured by a UV spectrophotometer (Shimadzu UV-2450 Spectrophotometer, Japan) at $670 \mathrm{~nm}$ and $470 \mathrm{~nm}$, respectively.

\subsubsection{Fatty acid profile determination}

In order to determine fatty acid profile of the olive oil samples, firstly methyl esterification reaction was carried out according to European Official Methods of Analysis (European Union Commission, 1991). After esterification reaction, the solution was vortexed and centrifuged in order to collect supernatant and then filtered into dark brown vials. Immediately after filtration, supernatant was injected into the gas chromatography (GC) device.

Fatty acid profiles of olive oil samples were examined by a GC (Agilent 6890, Agilent Technologies, USA) equipped with an autosampler (Agilent $7863 \&$ FID) and a split/splitless (1:50) injector. HP 88 capillary column (Agilent, USA) with dimensions of $100 \mathrm{~m}^{*} 0.25 \mathrm{~mm} \mathrm{ID}^{*} 0.2 \mu \mathrm{m}$ was used and helium with $2 \mathrm{~mL} / \mathrm{min}$ constant flow rate was selected as a carrier medium. Injection volume was $1 \mathrm{~mL}$ with the injection temperature of $250{ }^{\circ} \mathrm{C}$ while the detector temperature was kept at $280{ }^{\circ} \mathrm{C}$. Oven temperature was set to $120^{\circ} \mathrm{C}$ initially and was maintained there for $10 \mathrm{~min}$ then increased with a rate of $3{ }^{\circ} \mathrm{C} / \mathrm{min}$ until reaching to $220^{\circ} \mathrm{C}$ which was kept at this temperature for another $5 \mathrm{~min}$. FAME standard peaks were compared with sample chromatogram and the results were expressed as percentage of FAME.

\subsection{Fourier-transform infrared (FTIR) spectroscopy analysis}

All infrared spectra were recorded in mid-IR $\left(4000-650 \mathrm{~cm}^{-1}\right.$ wavenumber) range by a Perkin Elmer Spectrum 100 FTIR spectrometer (Perkin Elmer Inc., USA) having a deuterated tri-glycine sulphate (DTGS) detector. The instrument was equipped with a horizontal attenuated total reflectance (HATR) accessory with ZnSe 
crystal. For each spectrum, the number of scans was 64 while the resolution was set to $4 \mathrm{~cm}^{-1}$ and scan speed was $1 \mathrm{~cm} / \mathrm{s}$. Background spectra were collected before each measurement. Measurements were repeated two times.

\subsection{Statistical analysis}

All the statistical analyses were performed with SIMCA 13.0.3 software (Umetrics, Sweden). The need for multivariate evaluation exists due to chromatographic and spectroscopic methods' multivariate inheritance since more than one measurement can be made on a single sample (Brereton, 2003). In data analysis, whole FTIR spectra were used. Partial least squares (PLS) regression as the multivariate statistical analysis tool was applied for the prediction of chemical parameters from FTIR spectra.

PLS is a supervised regression method which aims at predicting $Y$ variables (fatty acid content including MUFA, PUFA, SFA, phenolic composition, TPC, chlorophyll and carotenoid content and oxidative stability) from $X$ variables (mid-IR spectra) by maximizing the correlation between them by a linear multivariate model (Eriksson, Kettaneh-Wold, Trygg, Wikström, \& Wold, 2006). In order to increase the predictive ability of the PLS model second derivative of FTIR profile which allows the elimination of noises and shifts was used. The derivative was calculated from moving quadratic submodels, each 15 data point long. The distance between each data point was 1 and edge effects were excluded.

As the validation technique, cross-validation method was used to assess how the models generalize to an independent data-set. Several parameters (root mean square error of calibration, RMSEC and cross-validation, RMSECV, regression coefficients for calibration, $\mathrm{R}_{\text {cal }}^{2}$ and cross-validation, $\mathrm{R}_{\mathrm{cv}}^{2}$ ) were also calculated to determine the predictive ability of the models. Regression coefficient provides an idea about the prediction efficiency and both calibration and validation $\mathrm{R}^{2}$ must be close to one for a good model (Bauer et al., 2008). RMSEC and RMSECV values are related with the error between measured value and predicted value at each calibration step and cross-validation step, respectively. It is expected that the differences between RMSEC and RMSECV values should be small and close to zero since each of these values is attributed to the error; therefore, the main idea of good prediction is the minimization of the error. Comparison of RMSEC and RMSECV values reveals whether the calibration model is over-fitted or not (Muik, Lendl, Molina-Díaz, Pérez-Villarejo, \& Ayora-Cañada, 2004). When evaluating the results of a prediction model all of these parameters must be taken into consideration. RMSECV value is calculated by SIMCA software. RMSEC is calculated according to equation given by Yucesoy and Ozen (2013).

\section{Results and discussions}

One of the multivariate statistical analysis tools, PLS regression was used to relate the mid-IR spectral data with the analytical results of several important chemical parameters of olive oils. Models were constructed for each response separately, only with the exception of chemically similar constituents; phenolic compounds (phenolic alcohols, flavonoids and phenolic acids) and fatty acids with PUFA, MUFA and SFA were used in a single model. Crossvalidation (leave one out) technique, which is generally the preferred method for medium-size data, was used to evaluate the model performance. To increase the efficiency of prediction one of the spectral filtering techniques, second derivative of the full spectra (4000-650 $\mathrm{cm}^{-1}$ wavenumber) was applied whereas unmodified spectral data of chlorophyll and carotenoid content, fatty acid profile and phenolic composition were used in oxidative stability prediction. Ranges and averages of the predicted parameters and statistical analysis results for constructed models are provided in Table 1. Measured ranges for the parameters correspond to typical values of olive oils.

\subsection{Oxidative stability}

OS values were predicted from FTIR spectral data by using PLS regression. The PLS model contains 5 principal components (PC) explaining $99 \%$ of the total variation (Fig. 1). Regression coefficient of calibration and cross-validation sets are determined as 0.99 and 0.81 , respectively and these values indicate good prediction (Table 1). RMSEC and RMSECV values are close to each other and also close to zero with the values of 0.11 and 0.86 , orderly. Slope of the calibration curve is equal to 1 accounting for high reliability.

In the literature, FTIR has been used to evaluate the freshness of olive oils under oxidative stress (Sinelli, Cosio, Gigliotti, \& Casiraghi, 2007). Direct determination of peroxide value (PV) was also investigated in two different studies (Bendini et al., 2007; Maggio et al., 2009). PV was predicted from FTIR spectra successfully with the application of a spectral filter (Maggio et al., 2009) whereas the result of the other study was not that promising (Bendini et al., 2007). There was only one study in the literature that used NIR spectroscopy for the determination of Rancimat generated OS and this study revealed promising results up to some extent (Mailer, 2004). In the present study; however, quantitative determination of OS from mid-IR spectra was investigated and it was found out that prediction results were quite satisfactory. To the best of our knowledge, Rancimat originated OS data is predicted from mid-IR spectroscopic measurement for the first time.

\subsection{Chlorophyll \& carotenoid content}

Chlorophyll and carotenoid contents of olive oils used in this study varied between 0.51 and $8.84 \mathrm{mg} / \mathrm{kg}$ oil and $0.11-25.63 \mathrm{mg} /$ $\mathrm{kg}$ oil, respectively. Chlorophyll and carotenoid values were predicted from FTIR profile and PLS regression curves are provided in Fig. 2. PLS models for chlorophyll and carotenoid content determination consist of 5 and 3 PCs, respectively. According to statistical results (Table 1 ), $\mathrm{R}^{2}$ value $(0.98)$ for chlorophyll calibration model is quite high while cross-validation $\mathrm{R}^{2}(0.69)$ is in the range of approximate prediction limits (0.66-0.80). RMSEC (0.18) and RMSECV (0.95) values are also good up to some degree. Slope of calibration curve (1) indicates a quite reliable prediction. However, carotenoid prediction parameters are not as good as chlorophyll due to the low value of regression coefficient of cross-validation (0.46) even though the value of calibration $R^{2}$ is high (0.95) meaning that the reproducibility of the model is low. Other parameters like RMSEC and RMSECV are relatively high and distant to each other. It can be concluded that prediction of chlorophyll content from FTIR data is successful while prediction of carotenoid is not as good as chlorophyll. In the literature, chlorophyll and carotenoid contents were also determined by different methods like chromatographic (Gandul-Rojas, Cepero, \& MínguezMosquera, 2000) and UV spectrophotometric methods (MínguezMosquera et al., 1991). As an IR method, NIR reflectance spectroscopy was used to determine chlorophyll content with high reliability $\left(\mathrm{R}^{2}=0.98\right)$ (Mailer, 2004); however, it is a new approach to predict chlorophyll and carotenoid contents from FTIR profile.

\subsection{Fatty acid profile}

The model of PLS regression for fatty acid profile of olive oils resulted with 4 PCs which explains $72.2 \%$ of total variation with a predictive ability of $45.2 \%$ in overall model. To see the prediction 
Table 1

Statistical results of the PLS regression models for the prediction of various compounds of olive oils from FTIR spectral data.

\begin{tabular}{|c|c|c|c|c|c|c|c|c|}
\hline Constituent & Mean & Range & PCs & $\mathrm{R}^{2}$ (cal.) & $\mathrm{R}^{2}(\mathrm{cv})$. & RMSEC & RMSECV & Regression equation \\
\hline $\mathrm{OS}^{1}(\mathrm{~h})$ & 1.72 & $0.10-4.41$ & 5 & 0.99 & 0.81 & 0.11 & 0.68 & $\mathrm{y}=\mathrm{x}+2.35^{*} 10^{-8}$ \\
\hline \multicolumn{9}{|c|}{ Colour Pigments (mg/kg) } \\
\hline $\mathrm{CHL}^{2}$ & 1.97 & $0.51-8.84$ & 5 & 0.98 & 0.69 & 0.18 & 0.95 & $\mathrm{y}=\mathrm{x}-1.04^{*} 10^{-7}$ \\
\hline $\mathrm{CRT}^{3}$ & 4.11 & $0.11-25.63$ & 3 & 0.95 & 0.46 & 0.93 & 3.01 & $\mathrm{y}=\mathrm{x}-5.10^{*} 10^{-8}$ \\
\hline \multicolumn{9}{|c|}{ Fatty acids (\%) } \\
\hline C $16: 0^{4}$ & 13.41 & $10.35-15.22$ & 4 & 0.87 & 0.70 & 0.35 & 0.55 & $\mathrm{y}=\mathrm{x}+2.21^{*} 10^{-7}$ \\
\hline C $16: 1^{5}$ & 0.80 & $0.13-1.42$ & 4 & 0.68 & 0.52 & 0.12 & 0.18 & $\mathrm{y}=0.97 * \mathrm{x}+0.03$ \\
\hline C $17: 0^{6}$ & 0.14 & $0.09-0.24$ & 4 & 0.74 & 0.05 & 0.02 & 0.03 & $\mathrm{y}=\mathrm{x}+3.83 * 10^{-9}$ \\
\hline$C 18: 0^{7}$ & 2.98 & $2.42-3.94$ & 4 & 0.61 & 0.35 & 0.24 & 0.31 & $\mathrm{y}=\mathrm{x}-1.33^{*} 10^{-7}$ \\
\hline $\mathrm{C} 18: 1 \mathrm{n} 9 \mathrm{c}^{8}$ & 68.88 & $65.66-76.59$ & 4 & 0.94 & 0.81 & 0.44 & 0.97 & $\mathrm{y}=\mathrm{x}-2.63^{*} 10^{-5}$ \\
\hline$C 18: 2 n 6 c^{9}$ & 11.99 & $4.90-15.13$ & 4 & 0.97 & 0.91 & 0.36 & 0.76 & $\mathrm{y}=\mathrm{x}-5.39 * 10^{-7}$ \\
\hline$C 20: 0^{10}$ & 0.46 & $0.34-0.63$ & 4 & 0.65 & 0.19 & 0.03 & 0.05 & $\mathrm{y}=\mathrm{x}-9.42^{*} 10^{-9}$ \\
\hline $\mathrm{C} 20: 1^{11}$ & 0.76 & $0.57-1.44$ & 4 & 0.39 & 0.23 & 0.11 & 0.12 & $\mathrm{y}=\mathrm{x}+1.70^{*} 10^{-8}$ \\
\hline $\mathrm{C} 18: 3 \mathrm{n}^{12}$ & 0.32 & $0.24-0.83$ & 4 & 0.09 & 0.00 & 0.08 & 0.08 & $\mathrm{y}=\mathrm{x}-9.52^{*} 10^{-8}$ \\
\hline $\mathrm{C} 22: 0^{13}$ & 0.12 & $0.09--0.23$ & 4 & 0.61 & 0.06 & 0.02 & 0.03 & $\mathrm{y}=\mathrm{x}-7.07^{*} 10^{-9}$ \\
\hline $\mathrm{SFA}^{14}$ & 17.32 & $13.51-19.93$ & 4 & 0.91 & 0.79 & 0.35 & 0.61 & $\mathrm{y}=\mathrm{x}-3.34^{*} 10^{-7}$ \\
\hline MUFA $^{15}$ & 70.66 & $66.91-78.61$ & 4 & 0.94 & 0.82 & 0.45 & 0.93 & $\mathrm{y}=\mathrm{x}-5.72^{*} 10^{-6}$ \\
\hline PUFA $^{16}$ & 12.02 & $4.90-15.82$ & 4 & 0.97 & 0.91 & 0.36 & 0.77 & $\mathrm{y}=\mathrm{x}+4.00^{*} 10^{-7}$ \\
\hline \multicolumn{9}{|c|}{ Phenolics (mg/kg) } \\
\hline $\mathrm{TPC}^{17}$ & 279.32 & $188.46-491.95$ & 5 & 0.99 & 0.74 & 6.06 & 45.26 & $\mathrm{y}=\mathrm{x}-5.18^{*} 10^{-6}$ \\
\hline Hxty $^{18}$ & 5.11 & $0.09-30.72$ & 6 & 0.97 & 0.68 & 1.02 & 4.66 & $\mathrm{y}=\mathrm{x}+1.75^{*} 10^{-7}$ \\
\hline Tyrs $^{19}$ & 11.07 & $0.73-44.19$ & 6 & 0.96 & 0.52 & 1.94 & 7.97 & $\mathrm{y}=\mathrm{x}+1.02^{*} 10^{-7}$ \\
\hline 4-Нypa ${ }^{20}$ & 0.74 & $0.14-5.99$ & 5 & 0.50 & 0.05 & 0.58 & 0.80 & $\mathrm{y}=\mathrm{x}-1.18^{*} 10^{-8}$ \\
\hline 3-Нypa 21 & 0.60 & $0.08-2.27$ & 5 & 0.59 & 0.08 & 0.24 & 0.40 & $\mathrm{y}=\mathrm{x}-4.61^{*} 10^{-8}$ \\
\hline $\mathrm{Vna}^{22}$ & 0.81 & $0.14-2.87$ & 5 & 0.77 & 0.26 & 0.23 & 0.41 & $\mathrm{y}=\mathrm{x}-1.68^{*} 10^{-8}$ \\
\hline $\mathrm{Sya}^{23}$ & 0.08 & $0.01-0.38$ & 5 & 0.63 & 0.19 & 0.03 & 0.06 & $\mathrm{y}=\mathrm{x}+5.95^{*} 10^{-9}$ \\
\hline $\mathrm{Cina}^{24}$ & 0.06 & $0.01-0.41$ & 5 & 0.69 & 0.19 & 0.04 & 0.07 & $y=x-3.64^{*} 10^{-9}$ \\
\hline $\mathrm{Cfa}^{25}$ & 0.10 & $0.01-0.60$ & 5 & 0.74 & 0.24 & 0.05 & 0.09 & $\mathrm{y}=\mathrm{x}-1.90 * 10^{-9}$ \\
\hline $\mathrm{Vnl}^{26}$ & 0.15 & $0.01-1.14$ & 8 & 0.97 & 0.31 & 0.03 & 0.16 & $\mathrm{y}=\mathrm{x}+2.26^{*} 10^{-9}$ \\
\hline $\mathrm{P}-\mathrm{cou}^{27}$ & 1.08 & $0.02-8.13$ & 5 & 0.82 & 0.36 & 0.54 & 1.06 & $\mathrm{y}=\mathrm{x}+3.03 * 10^{-8}$ \\
\hline Apig $^{28}$ & 1.14 & $0.04-5.29$ & 8 & 0.92 & 0.39 & 0.31 & 0.92 & $\mathrm{y}=\mathrm{x}-2.63^{*} 10^{-8}$ \\
\hline Lut $^{29}$ & 0.32 & $0.02-2.55$ & 8 & 0.96 & 0.08 & 0.10 & 0.52 & $\mathrm{y}=\mathrm{x}-1.00^{*} 10^{-9}$ \\
\hline
\end{tabular}

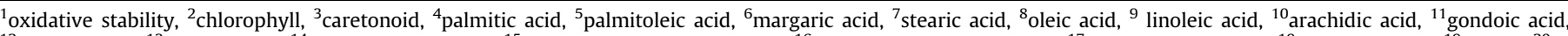

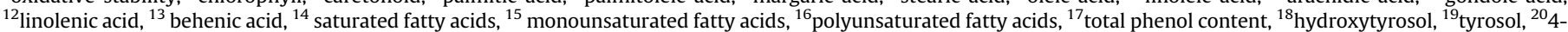

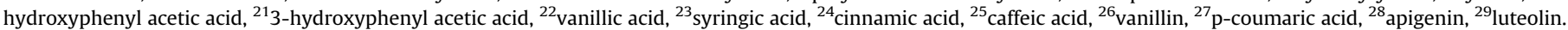

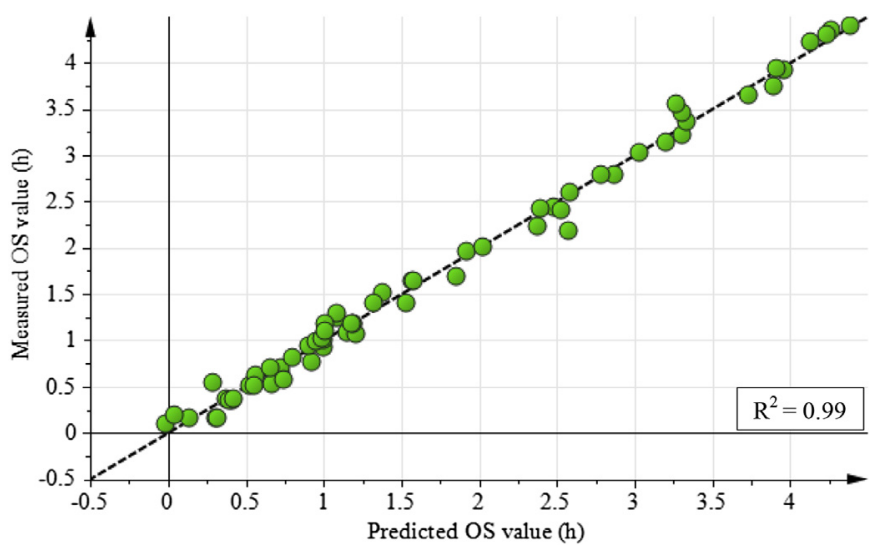

Fig. 1. Plot of measured vs predicted oxidative stability (OS) of olive oils obtained from PLS analysis of FTIR spectra.

power more clearly, each individual fatty acid components are analysed.

Firstly, the most abundant fatty acid component in olive oil, oleic acid (C18:1n9c), was investigated. Oleic acid was determined in the range of $65.7-76.6 \%$ in the present study. According to calibration model (Fig. 3a), $\mathrm{R}^{2}$ value of oleic acid was found as 0.94 which indicates good prediction of calibration set but it is not enough for ultimate conclusion. Cross validation technique was used to see the model validation and the result is quite successful with $R^{2}$ value of 0.81. RMSEC and RMSECV values were also found as 0.44 and 0.97 , respectively which are small and close to each other indicating that there is no over-fitting (Table 1). One of the important polyunsaturated fatty acids (PUFA), linoleic acid (C18:2n6c), was determined in the range of $4.90-15.13 \%$. The statistical values of the model are quite satisfactory (Table 1 ). Palmitic acid (C16:0) is the saturated fatty acid with the highest percentages in the olive oil samples. PLS model indicated that palmitic acid percentages could be detected with the regression coefficient value of 0.87 whereas cross-validation regression coefficient is 0.70 providing an approximate prediction on percentages of palmitic acid content. RMSEC and RMSECV values are close to each other and small $(0.35$ and 0.55 , orderly) as shown in Table 1.

MUFA, PUFA and SFA percentages were in the range of $66.91-78.61 \%, 4.90-15.82 \%, 13.51-19.93 \%$, respectively and they were predicted from FTIR data with the perfect $\mathrm{R}^{2}$ calibration values and the rest of the statistical parameters are also in the range of good prediction. In Fig. 3b, PLS regression plot for MUFA percentages are shown. For the rest of the fatty acids like palmitoleic (C16:1) and stearic (C18:0) acids, PLS results provide prediction to some extent whereas arachidic (C20:0), gondoic acid (C20:1), $\alpha$ linolenic (C18:3n3) and behenic (C22:0) acids do not have good prediction models. In summary, higher amount of fatty acids have higher $\mathrm{R}^{2}$ cal and $\mathrm{R}^{2}{ }_{\mathrm{cv}}$ while their RMSEC and RMSECV values are quite low. Oleic, linoleic and palmitic acids as individual fatty acids and MUFA, PUFA and SFA as combination of defined fatty acid groups could be predicted well from the FTIR data which is in good agreement with the findings of Galtier et al. (2008). Mailer (2004) also found out that fatty acids at high concentrations were predicted well on contrary to low concentration ones. As indicated in this study and also in the literature components having high concentrations could be predicted well from FTIR spectra. These 

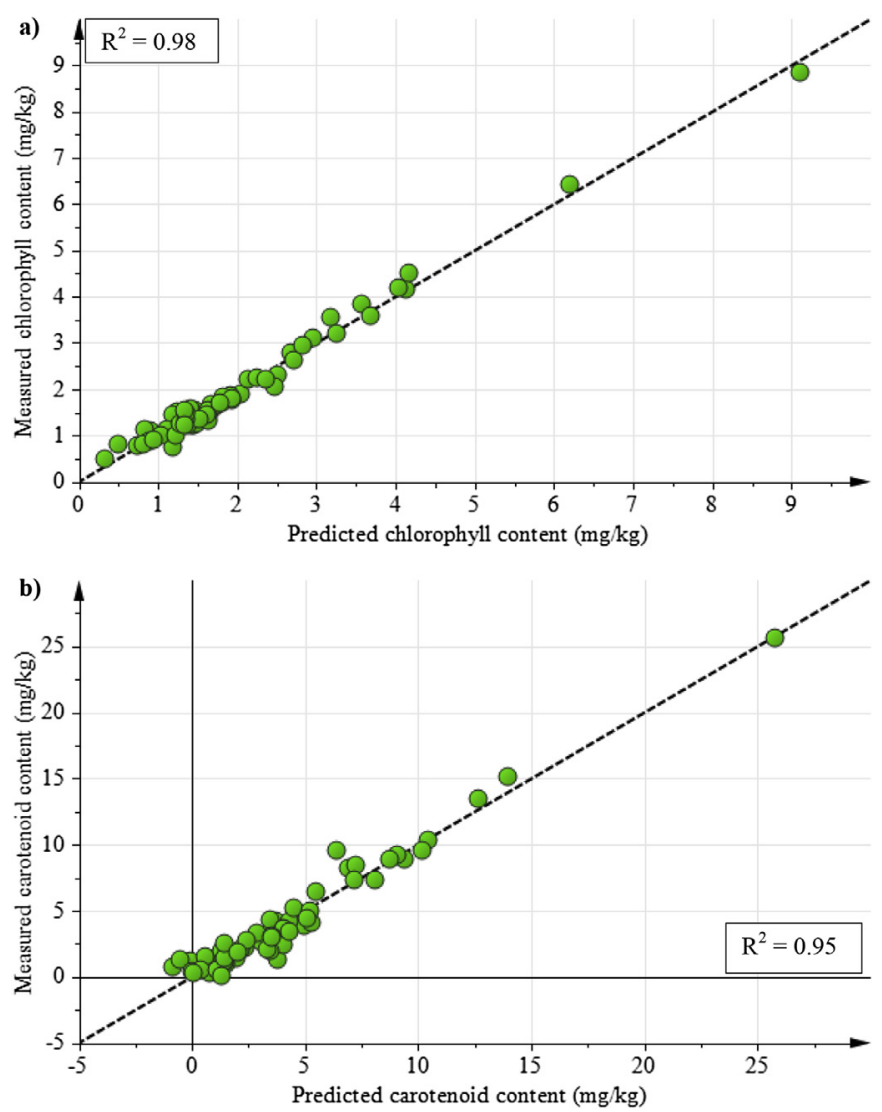

Fig. 2. Plot of measured vs predicted a) chlorophyll content $(\mathrm{mg} / \mathrm{kg}), \mathrm{b})$ carotenoid content $(\mathrm{mg} / \mathrm{kg})$ obtained from PLS analysis of FTIR spectra.

findings are also supported by the work of Gurdeniz, Ozen, and Tokatli (2010) in which stearic, oleic and linoleic acids were predicted quite well as in the present case. It was also reported successful predictions of stearic, arachidic and linolenic acids in the same study. All of these fatty acids individually and as a combination (MUFA, PUFA and SFA) have great importance in olive oil industry and each may also reveal authenticity of olive oil.

\subsection{Total phenolic content and individual phenolic compounds}

The PLS regression analysis using FTIR data for the prediction of phenolic compounds; phenolic alcohols, phenolic acids, and flavonoids and TPC of olive oils resulted in four different calibration models with 6 PCs which explain $96.9 \%$ of total variation with a moderate predictive ability of $61.2 \%$ in overall model, with 5 PCs explaining $67.8 \%$ of total variation with an insufficient prediction ability of $6.93 \%$, with 8 PCs including $95.3 \%$ explanation and low predictive ability (29.6\%) and 5 PCs explaining large variation (99\%) with confident prediction ability (73.8\%), respectively. To see the prediction power of FTIR spectra on each variable, PLS statistics for individual phenolic compounds are examined (Table 1). The best prediction among the phenolic compounds is observed for hydroxytyrosol with quite well $\mathrm{R}^{2}$ calibration value of 0.97 and $\mathrm{R}^{2}$ cross-validation value of 0.68 which indicates good validation of the model (Fig. 4a). This is also supported by the values of tolerable differences between RMSEC (1.02) and RMSECV (4.66). It is worth to emphasize that the good predictability of hydroxtyrosol content of olive oil is crucial due to its important contribution in olive oil oxidative stability (Carrasco-Pancorbo et al., 2005) and also its association with positive effect on health (Nan et al., 2014).
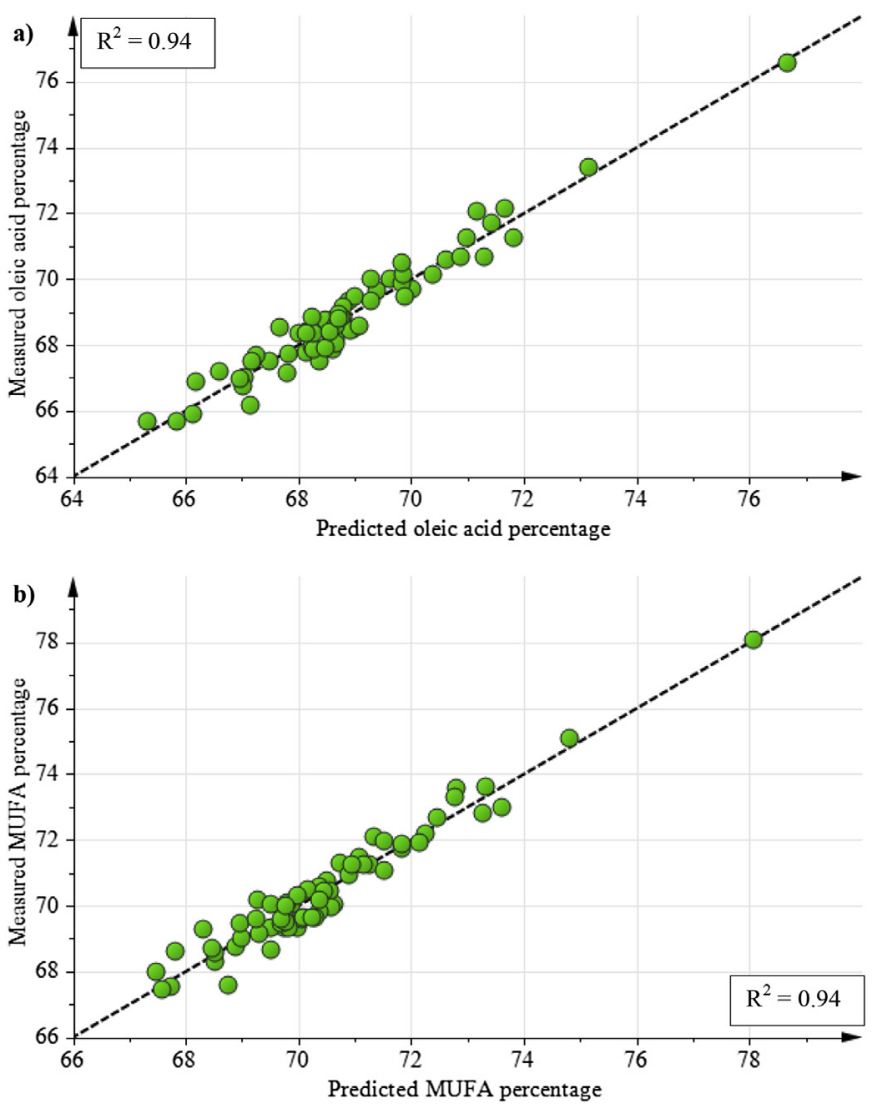

Fig. 3. Plot of measured vs predicted a) oleic acid (\%) and b) monounsaturated fatty acids (MUFA) (\%) obtained from PLS analysis from FTIR spectra.

Furthermore, certain health claims could be done on olive oil labels depending on the hydroxytyrosol content according to EFSA (European Food Safety Authority) (2011). Therefore, determination of hydroxtyrosol content of olive oils with FTIR spectroscopy in a shorter analysis time compared to chromatographic techniques would be beneficial for the industry. Besides hydroxtyrosol, another phenyl alcohol, tyrosol, was also predicted with $\mathrm{R}_{\text {cal }}^{2} 0.96$ and $\mathrm{R}^{2}{ }_{\mathrm{cv}}$ of 0.52 . Phenolic acids such as vanillic, cinnamic, caffeic and $\mathrm{p}-$ coumaric acids are not predicted as well as hydroxytyrosol and tyrosol due to the lower $\mathrm{R}^{2}$ cal values of $0.77,0.69,0.74$, and 0.82 , respectively. In addition, $R_{c v}^{2}$ values for these compounds are also low $(0.26,0.19,0.24$, and 0.36$)$. For the rest of the phenolic compounds, PLS models did not provide any predictions at all. Another phenolic compound group, flavonoids were also investigated and it was concluded that vanillin and apigenin were predicted with high calibration and average cross validation values (0.97, 0.92 and 0.31 , 0.39 ; orderly). TPC was also tried to be predicted from FTIR spectra (Fig. 4b) and the statistical values (Table 1) are quite promising with a high regression coefficient for calibration of 0.99 and the crossvalidation value (0.74). According to another study from the literature (Mailer, 2004), NIR spectroscopy achieved a marginal success in the determination of the TPC while Cerretani et al. (2010) obtained promising results for the prediction of total phenol with FTIR spectroscopy.

In the literature, IR spectra were used to determine TPC and phenolic compounds in olive fruit. Bellincontro et al. (2012) used near infrared (NIR) acousto optically tunable filter (AOTF) spectroscopy to determine TPC and some important phenolic compounds in olive fruit like oleuropein, verbascoside, and 3,4-DHPEAEDA. However, there is no study that determines the concentration 

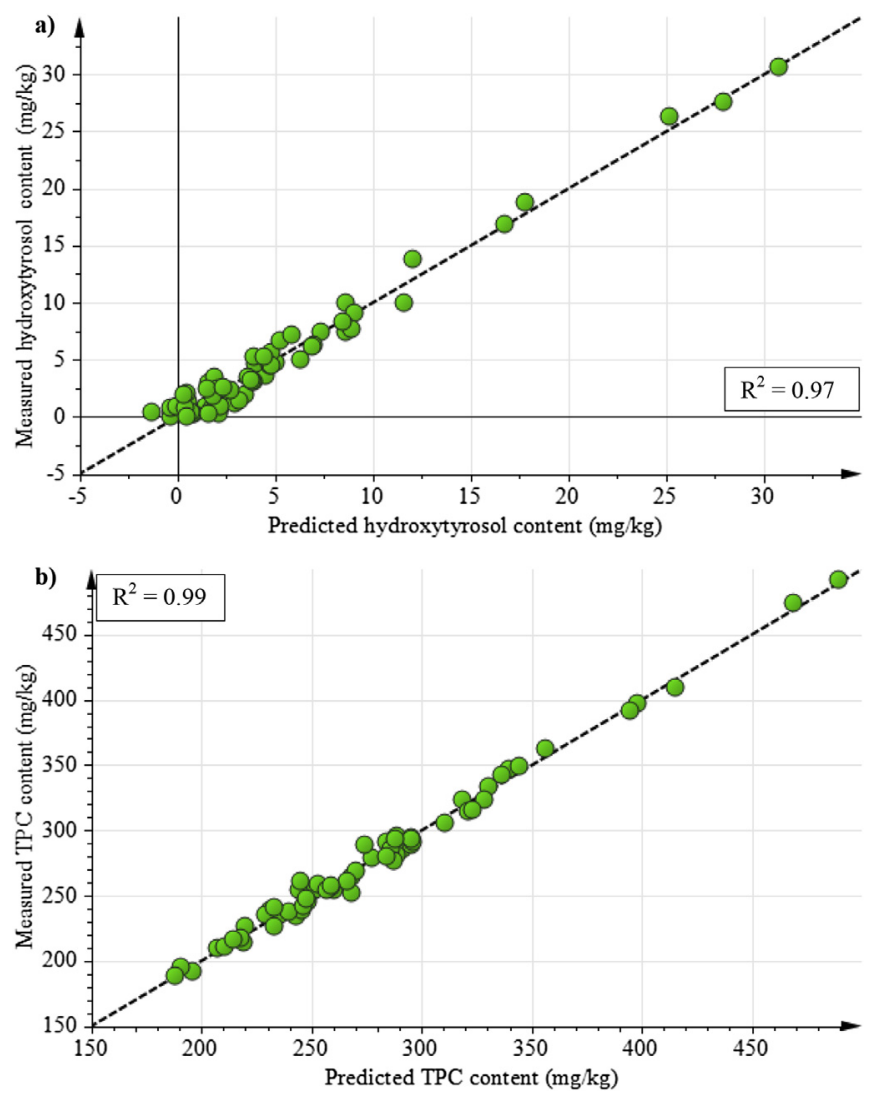

Fig. 4. Plot of measured vs predicted a) hydroxytyrosol ( $\mathrm{mg} / \mathrm{kg}$ ) and b) total phenol content (TPC) (mg/kg) obtained from PLS analysis from FTIR spectra.

of individual phenolic compounds in olive oil with FTIR spectroscopy in the literature. In the present study, it was aimed to find a correlation between mid-IR spectra and the content of phenolic compounds in olive oil, and good prediction results were observed for TPC and hydroxytyrosol content whereas tyrosol, vanillin and apigenin amounts were not predicted as good as TPC and hydroxytyrosol. For the rest of the phenolic compounds no significant results were observed.

\subsection{Prediction of oxidative stability from various chemical parameters}

In this part, the main aim is to observe the effect of individual components of fatty acids, phenolic substances, TPC, chlorophyll and carotenoid contribution on the OS of olive oils by using PLS regression and monitoring the variable influence on the projection (VIP) values; therefore, to find out any possible relation between overall chemical parameters (fatty acid, phenolic compounds, TPC, chlorophyll and carotene) and oxidative stability. Constructed PLS regression model explains $64 \%$ of the total variation with $13.7 \%$ predictive ability. $\mathrm{R}_{\text {cal }}^{2}(0.64)$ and $\mathrm{R}_{\mathrm{cv}}^{2}(0.14)$ provide slight prediction of OS from chemical data. Close RMSEC (0.77) and RMSECV (1.34) values indicate that there is no over fitting of the model. The reason of low prediction power could be the lack of other major oxidative stability contributors such as tocopherols which were not determined in the present study (Blekas, Tsimidou, \& Boskou, 1995).

Evaluation of VIP values gives an idea about the most important contributor to the oxidative stress and it is accepted that for a variable to be effective on the prediction, its VIP value should be higher or close to a threshold value of 1 . As a result of examination of VIP values, the most influential parameter for the oxidative stability of olive oils is determined as palmitic acid, with a VIP value of 1.83 (Fig. 5). Palmitic acid is the saturated fatty acid of the highest percentage in olive oil and is known for its stability against oxidative stress. For vanillic acid, cinnamic acid and hydroxytyrosol, similar VIP values are observed in the descending order of $1.56,1.51$, and 1.50, respectively (Fig. 5). According to Carrasco-Pancorbo et al. (2005), hydroxtyrosol has one of the highest antioxidant powers with other phenols like deacetoxy oleuropein aglycon and oleuropein aglycon. The present study also confirms the importance of hydroxtyrosol on oxidative stability with a VIP value of 1.50 . VIP values of palmitoleic and p-coumaric acids are close to each other with values of 1.40 and 1.29, respectively (Fig. 5). Caffeic acid, apigenin, tyrosol, gondoic acid, and total phenol content have VIP values in the descending order of 1.08, 1.03, 0.98, 0.96 and 0.92 and these values could be still considered as significant (Fig. 5). Rest of the parameters has lower VIP values and the variable effects become smaller and insignificant.

\section{Conclusion}

In this study, various chemical parameters, oxidative stability, chlorophyll and carotenoid content, fatty acid profile and phenolic composition of olive oils are estimated from FTIR spectra in combination with PLS analysis. Furthermore, OS is not only predicted from FTIR profile but also from combination of measured chemical parameters.

Prediction models for some fatty acids like oleic, linoleic, palmitic acids and MUFA, PUFA and SFA of olive oils were robust with higher $\mathrm{R}^{2}{ }_{\mathrm{cal}}, \mathrm{R}^{2}{ }_{\mathrm{cv}}$, and lower RMSEC and RMSECV. Oxidative stability and chlorophyll content were predicted perfectly while carotenoid content prediction is not as good as chlorophyll content determination using FTIR spectroscopy. PLS models of some phenolic compounds and TPC of olive oils from IR spectra were also examined and hydroxytyrosol and TPC were predicted promisingly. Apart from these, OS model developed from various chemical parameters (TPC, phenolic compounds, fatty acid content, chlorophyll, and carotenoid) provided an approximate prediction. The most significant contributors on oxidative stability of olive oils were determined as palmitic, vanillic and cinnamic acids,

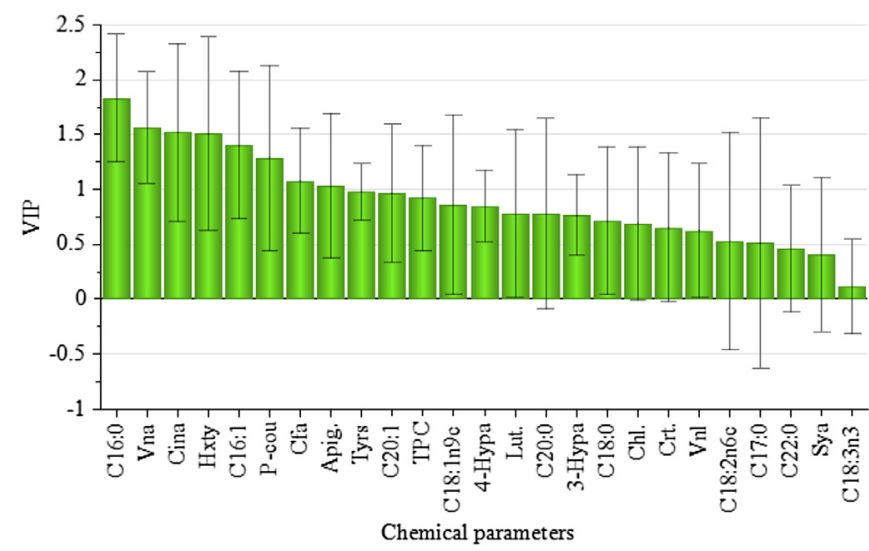

Fig. 5. Statistical results of the PLS regression model for the prediction of OS (h) from various chemical parameters (C16:0: palmitic acid, Vna: vanillic acid, Cina: cinammic acid, Hxty: hydroxytyrosol, C16:1: palmitoleic acid, P-cou: p-coumaric acid, Cfa: caffeic acid, Apig: apigenin, Tyrs: tyrosol, C20:1: gondoic acid, TPC: total phenol content, C18:1n9c: oleic acid, 4-Hypa: 4-hydroxyphenylacetic acid, Lut: luteolin, C20:0: arachidic acid, 3-Hypa: 3-hydroxyphenylacetic acid, C18:0: stearic acid, Chl: chlorophyll, Crt: carotenoid, Vnl: vanillin, C18:2n6c: linoleic acid, C17:0: heptadecanoic acid, C22:0: behenic acid, Sya: syringic acid, C18:3n3: linolenic acid). 
hydroxytyrosol, palmitoleic, p-coumaric and caffeic acids, apigenin, tyrosol, gondoic acid and TPC in decreasing importance in the studied case. To sum up, FTIR spectroscopy has high potential to predict the amount of some important chemical compositional and quality parameters of olive oils such as major fatty acids, some phenolic compounds (including TPC), oxidative stability and chlorophyll simultaneously in a short time with minimum chemical waste. The success of this and other similar studies in the literature indicates that FTIR in combination with chemometric techniques have potential of predicting other quality parameters of olive oil such as other oxidation indices (iodine value, peroxide value, anisidine value etc.), individual chlorophyll and carotene components, tocopherols, sterols and waxes. Rapid analyses of these chemical components would provide better control of quality during processing and storage and also allow in determining the authenticity of the product.

\section{Acknowledgements}

We would like to thank Biotechnology and Bioengineering Research Center and Environmental Research Center of Izmir Institute of Technology for their assistance in HPLC and GC analyses.

\section{References}

Allendorf, M., Subramanian, A., \& Rodriguez-Saona, L. (2012). Application of a handheld portable mid-infrared sensor for monitoring oil oxidative stability. Journal of the American Oil Chemists' Society, 89, 79-88.

Armenta, S., Garrigues, S., \& de la Guardia, M. (2007). Determination of edible oil parameters by near infrared spectrometry. Analytica Chimica Acta, 596, 330-337.

Bauer, R., Nieuwoudt, H., Bauer, F. F., Kossmann, J., Koch, K. R., \& Esbensen, K. H. (2008). FTIR spectroscopy for grape and wine analysis. Analytical Chemistry, 80, 1371-1379.

Bellincontro, A., Taticchi, A., Servili, M., Esposto, S., Farinelli, D., \& Mencarelli, F. (2012). Feasible application of a portable NIR-AOTF tool for on-field prediction of phenolic compounds during the ripening of olives for oil production. Journal of Agricultural and Food Chemistry, 60, 2665-2673.

Bendini, A., Cerretani, L., Di Virgilio, F., Belloni, P., Bonoli-Carbognin, M., \& Lercker, G. (2007). Preliminary evaluation of the application of the FTIR spectroscopy to control the geographic origin and quality of virgin olive oils. Journal of Food Quality, 30, 424-437.

Blekas, G., Tsimidou, M., \& Boskou, D. (1995). Contribution of $\alpha$-tocopherol to olive oil stability. Food Chemistry, 52, 289-294.

Brenes, M., García, A., García, P., Rios, J. J., \& Garrido, A. (1999). Phenolic compounds in Spanish olive oils. Journal of Agricultural and Food Chemistry, 47, 3535-3540.

Brereton, R. G. (2003). Chemometrics: Data analysis for the laboratory and chemical plant. UK: John Wiley \& Sons Ltd.

Carrasco-Pancorbo, A., Cerretani, L., Bendini, A., Segura-Carretero, A., Del Carlo, M., Gallina-Toschi, T., et al. (2005). Evaluation of the antioxidant capacity of individual phenolic compounds in virgin olive oil. Journal of Agricultural and Food Chemistry, 53, 8918-8925.

Cerretani, L., Giuliani, A., Maggio, R. M., Bendini, A., Gallina Toschi, T., \& Cichelli, A. (2010). Rapid FTIR determination of water, phenolics and antioxidant activity of olive oil. European Journal of Lipid Science and Technology, 112, 1150-1157.

Dong, W., Zhang, Y., Zhang, B., \& Wang, X. (2013). Rapid prediction of fatty acid composition of vegetable oil by Raman spectroscopy coupled with least squares support vector machines. Journal of Raman Spectroscopy, 44, 1739-1745.

EFSA. (2011). Panel on Dietetic Products, Nutrition and Allergies (NDA); Scientific Opinion on the substantiation of health claims related to polyphenols in olive and protection of LDL particles from oxidative damage (ID 1333, 1638, 1639, 1696, 2865), maintenance of normal blood HDL-cholesterol concentrations (ID 1639), maintenance of normal blood pressure (ID 3781), "anti-inflammatory properties" (ID 1882), “contributes to the upper respiratory tract health" (ID 3468), "can help to maintain a normal function of gastrointestinal tract" (3779), and "contributes to body defences against external agents" (ID 3467) pursuant to Article 13(1) of regulation (EC) No 1924/2006. EFSA Journal, 9, 2033-2058.

Eriksson, L., Kettaneh-Wold, N., Trygg, J., Wikström, C., \& Wold, S. (2006). Multi-and megavariate data analysis: Part I: Basic principles and applications. Umetrics.

European Union Commission. (1991). Regulation EEC 2568/91 on the characteristics of olive oil and olive-residue oil and on the relevant methods of analysis. Officia Journal of European Communities, L248.

Galtier, O., Le Dréau, Y., Ollivier, D., Kister, J., Artaud, J., \& Dupuy, N. (2008). Lipid compositions and French registered designations of origins of virgin olive oils predicted by chemometric analysis of mid-infrared spectra. Applied Spectroscopy, 62, 583-590.

Gandul-Rojas, B., Cepero, M. R. L., \& Mínguez-Mosquera, M. I. (2000). Use of chlorophyll and carotenoid pigment composition to determine authenticity of virgin olive oil. Journal of the American Oil Chemists' Society, 77, 853-858.

Gurdeniz, G., Ozen, B., \& Tokatli, F. (2010). Comparison of fatty acid profiles and mid-infrared spectral data for classification of olive oils. European Journal of Lipid Science and Technology, 112, 218-226.

Hidalgo, F. J., Gómez, G., Navarro, J. L., \& Zamora, R. (2002). Oil stability prediction by high-resolution 13C nuclear magnetic resonance spectroscopy. Journal of Agricultural and Food Chemistry, 50, 5825-5831.

Ismail, A. A., van de Voort, F. R., \& Sedman, J. (1997). Fourier transform infrared spectroscopy: principles and applications. In J. R. J. Paré, \& J. M. R. Bélanger (Eds.), Instrumental methods in food anaysis (pp. 93-139). Amsterdam: Elsevier (Chapter 4).

Lerma-García, M. J., Simó-Alfonso, E. F., Bendini, A., \& Cerretani, L. (2011). Rapic evaluation of oxidised fatty acid concentration in virgin olive oil using Fouriertransform infrared spectroscopy and multiple linear regression. Food Chemistry 124, 679-684.

Maggio, R. M., Kaufman, T. S., Carlo, M. D., Cerretani, L., Bendini, A., Cichelli, A., et al (2009). Monitoring of fatty acid composition in virgin olive oil by Fourier transformed infrared spectroscopy coupled with partial least squares. Food Chemistry, 114, 1549-1554.

Mailer, R. J. (2004). Rapid evaluation of olive oil quality by NIR reflectance spectroscopy. Journal of the American Oil Chemists' Society, 81, 823-827.

Matos, L. C., Cunha, S. C., Amaral, J. S., Pereira, J. A., Andrade, P. B., Seabra, R. M., et al (2007). Chemometric characterization of three varietal olive oils (Cvs. Cobrançosa, Madural and Verdeal Transmontana) extracted from olives with different maturation indices. Food Chemistry, 102, 406-414.

Mínguez-Mosquera, M. I. Rejano-Navarro, L., Gandul-Rojas, B., Sánchez Gómez, A. H., \& Garrido-Fernandez, J. (1991). Color-pigment correlation in virgin olive oil. Journal of the American Oil Chemists' Society, 68, 332-336.

Montedoro, G., Servili, M., Baldioli, M., \& Miniati, E. (1992). Simple and hydrolysable phenolic compounds in virgin olive oil. 1. Their extraction, separation, and quantitative and semiquantitative evaluation by HPLC. Journal of Agricultural and Food Chemistry, 40, 1571-1576.

Moros, J., Garrigues, S., \& de la Guardia, M. (2010). Vibrational spectroscopy provides a green tool for multi-component analysis. Trends in Analytical Chemistry, 29, 578-591.

Muik, B., Lendl, B., Molina-Díaz, A., Pérez-Villarejo, L., \& Ayora-Cañada, M. J. (2004) Determination of oil and water content in olive pomace using near infrared and Raman spectrometry. A comparative study. Analytical and Bioanalytical Chemistry, 379, 35-41.

Nan, J. N., Ververis, K., Bollu, S., Rodd, A. L., Swarup, O., \& Karagiannis, T. C. (2014) Biological effects of the olive polyphenol, hydroxytyrosol: an extra view from genome-wide transcriptome analysis. Hellenic Journal of Nuclear Medicine, 17 62-69.

Perona, J. S., Cabello-Moruno, R., \& Ruiz-Gutierrez, V. (2006). The role of virgin olive oil components in the modulation of endothelial function. The Journal of Nutritional Biochemistry, 17, 429-445.

Sinelli, N., Cosio, M. S., Gigliotti, C., \& Casiraghi, E. (2007). Preliminary study on application of mid infrared spectroscopy for the evaluation of the virgin olive oil "freshness". Analytica Chimica Acta, 598, 128-134.

Temime, S. B., Manai, H., Methenni, K., Baccouri, B., Abaza, L., Daoud, D., et al. (2008). Sterolic composition of Chétoui virgin olive oil: Influence of geographical origin. Food Chemistry, 110, 368-374.

Yucesoy, D., \& Ozen, B. (2013). Authentication of a Turkish traditional aniseed flavoured distilled spirit, raki. Food Chemistry, 141, 1461-1465. 